\title{
Novedades taxonómicas en Axonopus (Poaceae, Panicoideae, Paniceae) para Brasil
}

Taxonomic novelties in Axonopus (Poaceae, Panicoideae, Paniceae) for Brazil

\author{
Diego Giraldo-Cañas ${ }^{1}$
}

\begin{abstract}
Resumen
Axonopus P.Beauv. es un género nativo del continente americano, del que se conocen cerca de 72 especies, principalmente concentradas en el norte de Sudamérica, con algunos representantes aparentemente introducidos en el Viejo Mundo. Se reducen tres binomios a la sinonimia de especies previamente descritas de Brasil $(A$. villosus Swallen, A. obtusifolius (Raddi) Chase y A. pellitus (Nees ex Trin.) Hitchc. \& Chase), y se presenta el reconocimiento de la especie brasileña Axonopus kuhlmannii G.A.Black. Se presenta la distribución geográfica y ecológica, y se comentan algunas particularidades para los cuatro taxones estudiados.

Palabras clave: Flora de Brasil, gramíneas, neotrópico, taxonomía.
\end{abstract}

\begin{abstract}
Axonopus P.Beauv. is an American genus with ca. 72 species, mainly distributed in northern South America, with some species apparently introduced in the Old World. Three new synonyms of Axonopus are proposed for Brazil (A. villosus Swallen, A. obtusifolius (Raddi) Chase, and A. pellitus (Nees ex Trin.) Hitchc. \& Chase). Additionally, the name Axonopus kuhlmannii G.A.Black, endemic to Brazil, is accepted as distinct species. Ecological and geographical distribution plus comments on particular features are provided for the four species treated in the present study.
\end{abstract}

Key words: Flora of Brazil, grasses, Neotropics, taxonomy.

\section{Introducción}

Axonopus P.Beauv. pertenece a la tribu Paniceae de la subfamilia Panicoideae. Es un género nativo de las regiones tropicales y subtropicales de América, se distribuye desde el centro-sur de los Estados Unidos de América hasta la provincia de Buenos Aires (Argentina) y Chile (Isla de Pascua), así como en las islas del Caribe, con algunos representantes aparentemente introducidos en África, Australia, Asia tropical y en Portugal (Giraldo-Cañas 2008a). De este género se conocen cerca de 72 especies (Giraldo-Cañas 2008b), las cuales están mayormente concentradas en el norte de Sudamérica (Black 1963; Giraldo-Cañas 2008b). Sus especies crecen, principalmente, por debajo de los $1000 \mathrm{~m}$ de altitud, y constituyen importantes elementos en las sabanas naturales y en los afloramientos rocosos de los escudos precámbricos sudamericanos.

Este género incluye hierbas perennes, raras veces anuales, cespitosas, bajas a muy robustas, a veces rastreras, estoloníferas o rizomatosas, de hojas tiernas a muy duras y se distingue de otros miembros de la tribu Paniceae por presentar espiguillas solitarias en posición inversa, es decir, con la lema fértil abaxial respecto al raquis y por carecer de gluma inferior. Así, las características más contundentes para delimitar el género Axonopus son: espiguillas solitarias con gluma superior abaxial (posición inversa), ausencia de gluma inferior y reducción del antecio basal a la lema inferior estéril (Black 1963; Giraldo-Cañas 2008b).

Se presentan algunas novedades taxonómicas con el fin de aportar nuevos elementos para el esclarecimiento de la taxonomía y la sistemática del género Axonopus a nivel mundial, las cuales son producto de la continuación del estudio de este destacado género de Paniceae (Dedecca 1956; Black 1963; Renvoize 1984; Davidse 1987; Do Carmo Bastos 1991; Pohl \& Davidse 1994; Longhi-Wagner et al. 2001; Sousa da Rocha \& Secco 2004; GiraldoCañas 2000, 2001, 2002, 2003, 2008a, 2008b). Las novedades se detallan a continuación.

${ }^{1}$ Universidad Nacional de Colombia, Facultad de Ciencias, Instituto de Ciencias Naturales,Apartado 7495, Bogotá D. C., Colombia. dagiraldoc@unal.edu.co 


\section{Materiales y Métodos}

Se sigue el concepto morfológico de especie, con base en los postulados de Davis \& Heywood (1963), Crisci (1994) y Uribe Meléndez (2008), esto es, "una especie se define como un conjunto de individuos que presenta un espectro continuo de variación fenotípica y separado de otros conjuntos por discontinuidades morfológicas; en otras palabras, las especies son hipótesis acerca de la discontinuidad de la naturaleza". El estudio del género está basado en el análisis de las colecciones de los siguientes herbarios: AAU, AS, B, BA, BAA, BAF, BRG, CAUP, CEN, CEPEC, COAH, COL, CORD, CTES, F, FMB, G, HPUJ, HUA, HUQ, IAN, IBGE, K, LIL, LP, LPB, MA, MEDEL, MEXU, MO, NY, P, R, RB, RSA, SI, SP, TOLI, U, UPTC, US, VEN y XAL, abreviados de acuerdo con Holmgren et al. (1990). No obstante, el estudio detallado del material tipo depositado en US permitió proponer todos los sinónimos aquí destacados. Véanse Zuloaga et al. (2003) y Giraldo-Cañas (2008b) para la lista de otros sinónimos de las especies aquí tratadas. Cabe destacar que las palabras holotipo, isotipo, lectotipo, isolectotipo y otras similares, están escritas de acuerdo con Martínez-Laborde et al. (2002: xi), autores que adoptan y aconsejan la forma sin tilde.

\section{Resultados y Discusión}

Entre las novedades taxonómicas se destaca la reducción de tres binomios a la sinonimia de especies previamente descritas de Brasil y Venezuela, así como el reconocimiento de la especie brasileña Axonopus kuhlmannii G.A.Black. Estas novedades se detallan a continuación.

Axonopus comatus (Mez) Swallen, Fieldiana, Bot. 28(1): 21. 1951. Paspalum comatum Mez, Repert. Spec. Nov. Regni Veg. 15: 67. 1917. Tipo: BRASIL. RIO DE JANEIRO: A.F.M. Glaziou 15700 (holotipo B; isotipos BAA, K, US 2942140!).

Axonopus villosus Swallen, Fieldiana, Bot. 28(1): 21. 1951. Tipo: VENEZUELA. AMAZONAS: On summit of Cerro Duida, savanna hills, 10251200 m, 2.IX.1944, J. Steyermark 58226 (holotipo F; isotipo US 1911651!), syn. nov.

Axonopus comatus se distribuye en la Guayana venezolana (estados Amazonas y Bolívar), así como en el sudeste de Brasil, en donde sólo se conoce de la localidad tipo. Esta especie es frecuente en las praderas y los herbazales guayaneses venezolanos sobre turba en altiplanicies y áreas montanas, entre los 1000 y los $2200 \mathrm{~m}$. Merece destacarse, que si bien esta especie es frecuente en algunos ambientes guayaneses de Venezuela, su presencia en Brasil puede corresponder a una extinción, ya que sólo se conoce para la localidad tipo (Rio de Janeiro), situación evidenciable en los más de 1800 especímenes examinados de Axonopus, lo que no permitió detectar más ejemplares brasileños para A. comatus.

Después de estudiar los materiales tipo depositados en US de los dos binomios antes detallados, no se encontró diferencia alguna y por lo tanto, se propone la presente sinonimia. Anton (1982) ya había sugerido la afinidad entre Axonopus comatus y A. villosus, aunque no propuso la sinonimia respectiva.

Por otra parte, cabe destacar que Axonopus comatus es afín A. schultesii G.A.Black. No obstante, A. schultesii se diferencia principalmente por sus vainas glabras, por el mayor número de racimos por panoja (hasta 20 en $A$. schultesii vs. hasta 17 en $A$. comatus), generalmente por una mayor longitud de sus pedicelos $(0,3-1,8 \mathrm{~mm}$ de longitud, raramente hasta ca. $3 \mathrm{~mm}$ en $A$. schultesii vs. 0,5-1 mm en $A$. comatus), por sus espiguillas elipsoides de contorno lanceolado (ovoides en $A$. comatus), por su antecio superior elipsoide (ovoide en A. comatus), por la mayor relación del antecio superior respecto de su espiguilla $(0,2-1,4$ mm más corto que la espiguilla en $A$. schultesii vs. del mismo o hasta $0,4 \mathrm{~mm}$ más corto en $A$. comatus) y por el denso penacho en la región distal del antecio superior (penacho laxo en A. comatus).

Material adicional examinado: VENEZUELA. AMAZONAS: cerro Huachamacari, río Cunucunuma; Rim of NE Escarpment, 11.XII.1950, B. Maguire et al. 30148 (NY). BOLÍVAR: distrito Cedeño, sierra de Maigualida, sector NE, $5^{\circ} 33^{\prime} \mathrm{N}-65^{\circ} 13^{\prime} \mathrm{O}, 18 . \mathrm{XI} .1988$, O. Huber \& L. Izquierdo 12770 (SI, VEN).

Axonopus furcatus (Flüggé) Hitchc., Rhodora 8(95): 205. 1906. Paspalum furcatum Flüggé (como "Paspalus" furcatus), Gram. Monogr., Paspalum 114. 1810. Tipo: ESTADOS UNIDOS DE AMÉRICA. CAROLINAS: Bosc s.n. (holotipo no ubicado; isotipo US!).

Axonopus obtusifolius (Raddi) Chase, J. Wash. Acad. Sci. 13(9): 171. 1923. Paspalum obtusifolium Raddi, Agrostogr. Bras. 23. 1823. Tipo: BRASIL. GUANABARA: G. Raddi s.n. (holotipo PI; isotipos FI, US 2942535!), syn. nov. 
Esta especie se distribuye, de manera disyunta, en los Estados Unidos de América, Cuba, así como en Brasil. Está especie también ha sido citada para Argentina - como Axonopus obtusifolius - por Zuloaga et al. (2003), no obstante, nunca he encontrado ejemplares de dicho país a pesar de haber estudiado las colecciones de numerosos herbarios. Axonopus furcatus prefiere los suelos arenosos de tierras bajas y húmedas, y también se le encuentra en áreas degradadas y ambientes secundarios abiertos, entre el nivel del mar y los $1000 \mathrm{~m}$. Esta especie no está bajo ninguna amenaza evidente o palpable, pues sus poblaciones son numerosas y está presente en varios países.

Axonopus furcatus había sido tradicionalmente separada de $A$. obtusifolius únicamente por su distribución geográfica, siendo la primera reconocida exclusivamente para los Estados Unidos de América y Cuba, mientras que la segunda para Brasil. No obstante, y después de examinar el material tipo de ambos binomios así como una gran cantidad de especímenes, no se pudo advertir ninguna diferencia entre las mismas y por lo tanto, se propone la presente sinonimia. Anton (1982) ya había sugerido la afinidad entre A. furcatus y A. obtusifolius, aunque no propuso la sinonimia respectiva.

Material adicional examinado: BRASIL. MINAS GERAIS: Juiz de Fora, 20.II.1925, A. Chase 8539 (MO, US); 24.II.1925, A. Chase 8622 (MO, US). PARANÁ: Ypiranga, $P$. Dusén 3928 (R). RIO DE JANEIRO: Cabo Frio, II.1951, L. Mello 1088 (R). SANTA CATARINA: Rio Caveira, Biguaçu, 5 m alt., 11.II.1976, A. Bresolin 1194 (SI). Joinville, 3.I.1950, D. Hans 321 (R). SÃO PAULO: Jacareí, fazenda Santa Ana, 15.XII.1952, O. Boelcke 6787 (SI). Iguape, Morro das Pedras, XII.1917, A. Brade 7851 (R). CUBA. HABANA: Laguna de Ariguanabo, near Cayo La Rosa, 15.VIII.1921, E.L. Ekman 13105 (US), idem localidad, 17.VII.1923, E.L. Ekman 16928 (US). ESTADOS UNIDOS DE AMÉRICA. ALABAMA: Covington, 3.X.1971, R. Kral 44737(RSA). ARKANSAS: Along east side of Saline River at Old Saline River Bridge site, 29.IX.1984, R.D. Thomas et al. 91406 (RSA). CAROLINA DEL SUR: Orangeburg, 15.VIII.1905, A. Hitchcock 1566 (SI, US). FLORIDA: Flatwoods near Lake Hancock, 6.VI.1931, J.B. McFarlin 5676 (US). TEXAS: Fletcher, E.J. Palmer 10671 (MO).

Axonopus kuhlmannii G.A.Black, Advancing Frontiers Pl. Sci. 5: 156. 1963. Tipo: BRASIL. MATO GROSSO: Campo dos Urupás, ene 1919, J.C. Kuhlmann 1730 (holotipo IAN; isotipo US2236060!).

Especie endémica de Brasil, de la que sólo se conocen unos pocos ejemplares de Guaporé (Rondônia) y Mato Grosso. Dado que sólo se conocen tres colecciones de Axonopus kuhlmannii, y además éstas son antiguas, se puede inferir que la especie está extinta de la naturaleza.

A esta especie no se le había reconocido ningún estatus formal en el reciente catálogo de las gramíneas del Nuevo Mundo (véase Zuloaga et al. 2003: 126), aunque desafortunadamente dichos autores (Zuloaga et al. 2003) nunca especificaron ni dejaron en claro el por qué no le conferían ningún estatus de reconocimiento a esta especie. Cuando Black (1963) la propuso como nueva especie, la ubicó en Axonopus serie Fastigiati G.A.Black, una serie endémica de Brasil, la cual está compuesta por dos especies $(A$. fastigiatus (Nees ex Trin.) Kuhlm. y $A$. kuhlmannii). Esta serie está ubicada en la sección Axonopus, que Black (1963: 154) la definió por los siguientes caracteres: plantas perennes, raquis escabroso, glumas pilosas y/o ciliadas y antecio superior pajizo a castaño.

Cabe destacar que la especie Axonopus kuhlmannii no fue tratada ni mencionada en estudios o listas posteriores a Black (1963) (e.g. Anton 1982; Renvoize 1984; Dubs 1998; Longhi-Wagner et al. 2001; Sousa da Rocha \& Secco 2004; De Oliveira et al. 2009; Filgueiras 2010). Por otra parte, nunca he observado especímenes de Axonopus que hayan sido determinados como A. kuhlmannii, a pesar de haber examinado más de 1800 ejemplares de este género en 43 herbarios del mundo (e.g. AAU, AS, B, BA, BAA, BAF, BRG, CAUP, CEN, CEPEC, COAH, COL, CORD, CTES, F, FMB, G, HPUJ, HUA, HUQ, IAN, IBGE, K, LIL, LP, LPB, MA, MEDEL, MEXU, MO, NY, P, R, RB, RSA, SI, SP, TOLI, U, UPTC, US, VEN y XAL). Las características que diferencian las dos especies de la serie Fastigiati se datallan en la Tabla 1.

Además de las características antes señaladas (Tabla 1), Axonopus kuhlmannii es una especie muy peculiar, ya que es la única en el género que posee la gluma superior con los nervios laterales fuertemente escabrosos, y además, presenta el antecio superior castaño con su extremo distal pajizo. Estas dos características la hacen fácilmente reconocible, no sólo en la serie Fastigiati sino entre todas las especies del género. Desafortunadamente, sólo fue posible examinar el isotipo; no obstante, las particularidades de esta especie permiten reconocerla y aceptarla como entidad distinta y no hay posibilidad de confundirla ni con su especie más cercana (A. fastigiatus) (Tabla 1) ni con las demás especies de Axonopus (véase Giraldo-Cañas 2008b). 
Axonopus siccus (Nees) Kuhlm., Com. Linh. Telegr., Bot. 11: 87. 1922. Paspalum siccum Nees, como Paspalum siccus, Fl. bras. Enum. Pl.: 28. 1829. Tipo: BRASIL. MINAS GERAIS: in districtu Adamantum, Martius s.n. (holotipo M; isotipo US 80020!).

Axonopus pellitus (Nees ex Trin.) Hitchc. \& Chase, Contr. U.S. Natl. Herb. 18(7): 301. 1917. Paspalum pellitum Nees ex Trin., Gram. Panic. 89. 1826. Tipo: BRASIL. Sello s.n. (holotipo LE-TRIN; isotipos B, BAA, US 1258173!, US2 855755!, US 2942521!), syn. nov.

Esta especie se distribuye en Argentina (norte), Bolivia, Brasil, Paraguay y Uruguay. Axonopus siccus crece en campos, sabanas de diferente tipo, en arbustales, en escarpes rocosos, en grietas de afloramientos rocosos, en bordes de bosque y en zonas alteradas, tanto de zonas bajas como de zonas montañosas. Esta especie presenta un amplio rango altitudinal, desde los 100 hasta los $2800 \mathrm{~m}$. Axonopus siccus es una especie muy frecuente, ampliamente distribuida y además, forma matas densas en variados ambientes, lo que unido a la apreciable cantidad de colecciones - tanto antiguas como recientes - hace que no esté bajo ninguna categoría de amenaza crítica.

A raíz de su amplia distribución latitudinal y altitudinal, se han propuesto varios binomios, los cuales corresponden a meras variantes locales. Esta situación es corroborada con el análisis de una gran cantidad de especímenes, entre ellos el estudio detallado de material tipo, lo que permitió proponer esta nueva sinonimia para $A$. siccus en el presente estudio.

Axonopus siccus, al igual que varias de las especies del género, presenta una gran variación morfológica, la cual se verificó en la gran cantidad de ejemplares examinados. Esta variación podría ser derivada de la gran amplitud ecológica de la especie, representada principalmente en la gama de formaciones vegetales en que está presente y en su amplio espectro altitudinal. La variación morfológica se presenta principalmente en la pilosidad de las hojas y las espiguillas, dimensiones de la planta y espiguillas y en la forma de las hojas. Por lo regular, las poblaciones brasileñas de $A$. siccus son las más pubescentes.

Tabla 1 - Características de las especies de Axonopus P.Beauv. serie Fastigiati G.A.Black. Table 1 - Features of the species of Axonopus P.Beauv. series Fastigiati G.A.Black.

\begin{tabular}{|c|c|c|}
\hline Característica & $\begin{array}{l}\text { Axonopus fastigiatus } \\
\text { (Nees ex Trin.) Kuhlm. }\end{array}$ & $\begin{array}{l}\text { Axonopus kuhlmannii } \\
\text { G.A.Black }\end{array}$ \\
\hline Número de racimos por panoja & 1-2 (usualmente 2 conjugados) & 3-10 (alternos) \\
\hline Raquis & $\begin{array}{l}\text { Recto, } 0,5-1 \mathrm{~mm} \text { de ancho, } \\
10-15 \text { espiguillas por } 25 \mathrm{~mm}\end{array}$ & $\begin{array}{l}\text { Flexuoso, } 0,6-0,7 \mathrm{~mm} \text { de ancho, } \\
17-25 \text { espiguillas por } 25 \mathrm{~mm}\end{array}$ \\
\hline Espiguillas & $\begin{array}{l}\text { Elípticas, acuminadas, } \\
(2,5) 3,2-4,5 \mathrm{~mm} \text { long. }\end{array}$ & Oblongas, agudas, 1,9-2,1 mm long. \\
\hline Gluma superior & $\begin{array}{l}\text { 2-4-nervia, largamente ciliada, } \\
\text { nervios laterales escabriúsculos }\end{array}$ & $\begin{array}{l}\text { 3-5-nervia, cortamente pilosa, no ciliada, } \\
\text { nervios laterales fuertemente escabrosos }\end{array}$ \\
\hline Antecio superior & $\begin{array}{l}3 \mathrm{~mm} \text { long., acuminado, } \\
\text { el ápice piloso, pajizo }\end{array}$ & $\begin{array}{l}\text { 1,5 mm long., obtuso, glabro, } \\
\text { castaño con la porcion distal pajiza }\end{array}$ \\
\hline Distribución & $\begin{array}{l}\text { Áreas montanas de Brasil } \\
\text { (Bahia, Distrito Federal, Goiás, } \\
\text { Minas Gerais y São Paulo), } \\
\text { entre los } 600 \text { y los } 1300 \text { m de altitud }\end{array}$ & $\begin{array}{l}\text { Sólo conocida de dos localidades de } \\
\text { Rondônia y de una de Mato Grosso }\end{array}$ \\
\hline Ejemplares examinados & $\begin{array}{l}\text { Bahia: M. Aparecida da Silva et al. } 1586 \text { (SI). } \\
\text { Distrito Federal: H. Irwin \& T. Soderstrom } \\
5214 \text { (MO), H. Irwin \& T. Soderstrom } 5792 \\
\text { (NY, SI). Minas Gerais: G. Black \& } \\
\text { M. Magalhães } 51-11805 \text { (COL), Y. Mexia } \\
5880 \text { (MO, R), F. Zuloaga \& O. Morrone } \\
\text { 4693, 4699, } 4702 \text { (SI) }\end{array}$ & Mato Grosso: J.C. Kuhlmann 1730 (US) \\
\hline
\end{tabular}


Material adicional examinado: ARGENTINA. CORRIENTES: depto. Empedrado, Tres Marías, ca. al río Paraná, 20.III.1998, A. Schinini 34411 (CTES, SI). JUJUY: depto. Tumbaya, Chilcayo, finca del Dr. Gronda, 5.II.1995, N. Deginani et al. 377 (SI). MISIONES: depto. San Ignacio, parque provincial Teyucuaré, 30.IX.1998,D. Giraldo-Cañas \& F. Biganzoli 2802, 2803 (SI). BOLIVIA. LA PAZ: prov. Nor Yungas, arriba de Coroico, 25.II.1990, S. Beck 17469 (LPB, SI, US). SANTA CRUZ: Nuflo de Chávez, estación Las Madres, 20.III.1986, T. Killeen 1839 (F, SI, US). TARIJA: prov. Arce, entre Padcaya y Cañas, 29.I.1988, S. Beck 16255 (LPB, SI). BRASIL. BAHIA: serra do Sincorá, on Ibicora road, by the Río Preto, 2.II.1974, R. Harley 15863 (CEPEC, K). Palmeiras, morro do Pai Inacio, 11.II.1994, F. Zuloaga et al. 4780 (SI). DISTRITO FEDERAL: fazenda Água Limpa, 9.I.1990, D. Alvarenga \& F. Oliveira 584 (IBGE, SI). Sobradinho, 19.II.1992, T. Filgueiras \& F. Zuloaga 2021 (IBGE, SI). Chapada da Contagem, ca. $10 \mathrm{~km}$ E of Brasília, 12.I.1966, H. Irwin et al. 11583 (COL, NY, US). GOIÁS: São Gabriel, campo de Murundum, 20.II.1992, T. Filgueiras \& F. Zuloaga 2039 (IBGE, SI). Alto Paraíso de Goiás, 7 km $\mathrm{N}$ de Alto Paraiso, 21.II.1992, T. Filgueiras \& F. Zuloaga 2070 (IBGE, SI). Cristalina, 26.II.1992, T. Filgueiras \& F. Zuloaga 2170 (IBGE, SI). Serra dos Pireneus, Pirenópolis, 14.I.1972, H. Irwin et al. 34088 (COL, US); 18.I.1972, H. Irwin et al. 34526 (MO, NY, RSA, US). MATO GROSSO: between Campo Grande and Dourados, 14-17.II.1930, A. Chase 10903 (MO, US). Xavantina; 3.IV.1968, J. Ratter et al. 808 (K, NY). MINAS GERAIS: serra do Espinhaço, serra do Cipó, 18.II.1972, W. Anderson et al. 36258 (NY, RSA, US). Itacolomi, 8.IV.1925, A. Chase 9386 (NY, US); 30.I.1971, H. Irwin et al. 29369 (RSA). Serra do Curral, SE of Bello Horizonte, 19.III.1925, A. Chase 8920 (F, MO, US). Lagoa Santa, $42 \mathrm{~km}$ N of Bello Horizonte, 23-24.III.1925, A. Chase 9022 (MO, US). Hargreaves, 21-22.XII.1929, A. Chase 10241 (MO, US). Serra do Espinhaço, road to Salinas, 11.II.1969, H. Irwin et al. 23104 (RSA, US). Serra do Cabral, 9.III.1970, H. Irwin et al. 27281 (COL, NY, US). Parque Nacional do Caparaó, 1.IV.1989, P. Krieger 23575 (COL). Rodovia de Cardeal Mota a Conceição do Mato Dentro, BR010, 17.II.1993, F. Zuloaga \& O. Morrone 4566, 4570, 4582, 4595 (SI). BR-367, 3 km al N de Madanha, 19.II.1993, $F$. Zuloaga \& O. Morrone 4643, 4645 (SI). 15 km de Curvelo a Cordisburgo, 19.II.1993, F. Zuloaga \& O. Morrone 4659 (SI); Sierra da Piedade, Santuario, 21.II.1993, F. Zuloaga \& O. Morrone 4673 (SI). PARANÁ: $13 \mathrm{~km}$ NE of Atuba along highway 116; 9.III.1976, G. Davidse et al. 10966 (COL, MO); Virmond, BR-277 to Curitiba, 14.III.1976, G. Davidse et al. 11285 (COL); Guarapuava along highway BR-277 15.III.1976, G. Davidse et al. 11311 (COL). Castro along highway PR-11, 15.III.1976, G. Davidse et al. 11390 (MO); Pinhaes, 12.II.1914, P. Dusén 14502 (SI, US). SANTA CATARINA: Lajes, 18.II.1958, J. Anattos 5197 (SI, US); Entre Irani e Ponta Serrada, 27.II.1964, A. Castellanos 24605 (COL). SÃOPAULO: Campos do Jordão, serra Mantiqueira, 20-22.V.1925, A. Chase 9858 (paratipo de A. monticola, MO); Sin localidad precisa, nos campos da serra do Itatiaia, 1900 m, III.1894, E. Ule 242 (R). PARAGUAY.
AMAMBAY: Sierra de Amambay, años 1907-1908, E. Hassler 10171 (LIL). Parque Nacional Cerro Corá, próximo al río Aquidabán Niguií, 30.IV.1992, O. Morrone \& J. Pensiero 467 (SI); Camino a colonia Naranjaí, 2.V.1992, O. Morrone \& J. Pensiero 522 (SI); III.1934, T. Rojas 6770 (BAA); IV.1934, T. Rojas 6826(BAA). CAAGUAZÚ: ruta a Oviedo, 2.III.1950, B. Rosengurtt B-5871 (BAA). CENTRAL: in regione lacus Ypacaray, II.1913, E. Hassler 11548 (G, MO, US). CORDILLERA: Piribebuy, salto Piraretá, III.1942, Pavetri (?) \& T. Rojas 9519 (BAA). San Bernardino, río Salado, II.1915, T. Rojas 1037 (BAA, US). Tobatí, cerro Cabayú, I.1928, T. Rojas 5259 (BAA). GUAIRÁ: colonia independencia, cerro Pelado; III.1924, T. Rojas 4822 (BAA). MISIONES: San Ignacio, 16.II.1950, B. Rosengurtt B-5780 (LIL). SAN PEDRO: San Estanislao, estancia La Manina, 13.II.1975, T. Pedersen 11051 (SI). URUGUAY. RIVERA: On main road from Montevideo to Rivera, near Paso Empedrado, 21.III.1984, T. Pedersen 13880 (SI). Batoví, 8.III.1991, T. Pedersen 15694 (SI). Cuchilla Cuñapirú, ruta 29, 28.I.1958, B. Rosengurtt B7029 (SI).

\section{Agradecimientos}

Quiero manifestar mi profundo agradecimiento a la Universidad Nacional de Colombia y a su Instituto de Ciencias Naturales (COL) (Bogotá D.C., Colombia), al Herbario Nacional de los Estados Unidos de América (US), al Smithsonian Institution, Washington D.C., EE.UU. y al Instituto de Botánica Darwinion (SI), Buenos Aires, Argentina, por las facilidades permanentes para llevar a cabo los estudios en gramíneas. El Smithsonian Institution, así como el Herbario Nacional de los Estados Unidos de América, financiaron el viaje y la estadía del autor en Washington D.C. Quiero expresar mi eterno sentimiento de gratitud a los doctores F.O. Zuloaga (SI), O. Morrone (SI), P.M. Peterson (US), R. Soreng (US), L. Giussani (SI), S. Renvoize (K), J.F. Veldkamp (L) y S. Lægaard (AAU), por su permanente, grata y valiosa colaboración en los estudios agrostológicos, así como por sus valiosos comentarios. A los dos evaluadores anónimos por sus valiosos comentarios y correcciones. Al cuerpo editorial de la Revista Rodriguésia por su valiosa colaboración. Esta contribución es derivada del proyecto "Estudios sistemáticos en gramíneas de Colombia. Parte II" de la Universidad Nacional de Colombia, sede Bogotá D.C.

\section{Literatura}

Anton, A. 1982. Las especies de Axonopus sección Axonopus: sinopsis morfológica y taxonómica. Tesis Doctoral. Universidad Nacional de Córdoba, Córdoba. 228p.

Black, G.A. 1963. Grasses of the genus Axonopus (a taxonomic treatment). Advancing Frontiers in Plant Science 5: $1-186$. 
Crisci, J. 1994. La especie: realidad y conceptos. In: Llorente Bousquets, J. \& Luna, I. (orgs.). Taxonomía biológica. Universidad Autónoma de México-Fondo de Cultura Económica, México D.F. Pp. 53-64.

Davidse, G. 1987. Four new species of Axonopus (Poaceae: Paniceae) from tropical America. Annals of the Missouri Botanical Garden 74: 416-423.

Davis, P.H. \& Heywood, V.H. 1963. Principles of angiosperm taxonomy. Oliver \& Boyd, Edinburgh \& London. 190p.

Dedecca, D. 1956. As espécies brasileiras do gênero Axonopus (Gramineae). Bragantia 15: 251-296.

De Oliveira, R.P.; Longhi-Wagner, H.M.; Filgueiras, T.S.; Costa da Mota, A. \& Viana, P.L. 2009. Poaceae. In: Lima Santos, I. (ed.). Plantas raras do Brasil. Conservação Internacional-Universidade Estadual de Feira de Santana, Belo Horizonte. Pp. 326-340.

Do Carmo Bastos, M. 1991. A flora rupestre da serra de Carajás (Gramineae). II. Gênero Axonopus P. Beauv. Boletim do Museu Paraense Emílio Goeldi, sér. Bot. 7: 473-483.

Dubs, B. 1998. Prodromus Florae Matogrossensis. Part I. Checklist of Angiosperms \& Part II. Types from Mato Grosso. Betrona Verlag, Küsnacht. 444p.

Filgueiras, T.S. 2010. Poaceae-Axonopus. In: Lista de espécies da flora do Brasil. Disponível em <http:// floradobrasil.jbrj.gov.br/2010>. Acesso em 18 março 2010.

Giraldo-Cañas, D. 2000. Una nueva sección del género Axonopus (Poaceae, Panicoideae, Paniceae). Revista de la Academia Colombina de Ciencias Exactas, Físcas y Naturales 24: 183-191.

Giraldo-Cañas, D. 2001. Sinopsis de la sección Cabrera del género neotropical Axonopus (Poaceae, Panicoideae, Paniceae). Revista de la Academia Colombina de Ciencias Exactas, Físcas y Naturales 25: 207-223.

Giraldo-Cañas, D. 2002. Las especies de la sect. Lappagopsis del género neotropical Axonopus (Poaceae, Panicoideae, Paniceae). Revista de la Academia Colombina de Ciencias Exactas, Físcas y Naturales 26: 13-23.
Giraldo-Cañas, D. 2003. Revisión de las especies del género neotropical Axonopus P.Beauv. (Poaceae: Panicoideae: Paniceae), serie Barbigeri. Tesis Doctoral. Universidad Nacional de La Plata, La Plata, Buenos Aires. 514p.

Giraldo-Cañas, D. 2008a. Revisión del género Axonopus (Poaceae: Paniceae): Primer registro del género en Europa y novedades taxonómicas. Caldasia 30: 301-314.

Giraldo-Cañas, D. 2008b. Sistemática del género Axonopus (Poaceae: Panicoideae: Paniceae) y revisión de las especies de la serie Barbigeri. Serie Biblioteca José Jerónimo Triana 17: 1-211.

Holmgren, P.; Holmgren, N. \& Barnett, L. 1990. Index Herbariorum. Part I: The herbaria of the world. The New York Botanical Garden, New York. 693p.

Longhi-Wagner, H.M.; Bittich, V.; Wanderley, M.G.L. \& Shepherd, G.J. 2001. Poaceae. Flora fanerogamica do estado de São Paulo. Instituto de Botânica, São Paulo. Vol. 1. Pp. 1-292.

Martínez-Laborde, J.B.; Chiang, F. \& Kiesling, R. 2002. Presentación a la edición en español. In: Kiesling, R. (ed.). Código Internacional de Nomenclatura Botánica (Código de Saint Louis). Edición en español, Instituto de Botánica Darwinion-Missouri Botanical Garden, Buenos Aires. Págs. ix-xiv.

Pohl, R. \& Davidse, G. 1994. Axonopus. In: Flora Mesoamericana. Vol. 6. Pp. 356-359.

Renvoize, S. 1984. The grasses of Bahia. Royal Botanic Gardens, Kew. 301p.

Sousa da Rocha, A. \& Secco, R. 2004. Contribuição à taxonomia de Axonopus P.Beauv. (Poaceae) no estado do Pará, Brasil. Acta Botanica Brasilica 18: 295-304.

Uribe Meléndez, J. 2008. Monografía de Frullania subgénero Meteoriopsis (Frullaniaceae, Marchantiophyta). Caldasia 30: 49-94.

Zuloaga, F.; Morrone, O.; Davidse, G.; Filgueiras, T.; Peterson, P.; Soreng, R. \& Judziewicz, E. 2003. Catalogue of New World grasses (Poaceae): III. Subfamilies Panicoideae, Aristidoideae, Arundinoideae, and Danthonioideae. Contributions from the United States National Herbarium 46: 1-662. 